recent experiment with scattered electrons at the Stanford Linear Accelerator, an asymmetry in agreement with theory was found. New experiments now underway could solve this problem within a few months.

It became very clear, that the more traditional spectroscopic and interference spectroscopic methods have, of late, been stimulated by new techniques and problems. Thus research in astrophysics has recently raised the need for more "spectra of highly stripped ions" which can now be generated in laser produced plasmas as reported by U. Feldman. W.R.S. Garton's research work in which, by means of short wavelength synchrotron radiation, spectra of inner and doubly excited electrons are obtained and investigated, also shows clearly the importance of true spectroscopy for progress in atomic physics. This is just one reason for extending classical level classification work and completing the spectra so far only partly known. This work is obviously profiting from more and more powerful computer programmes.

Generally it was felt and emphasized in round table discussions that there again exists a definite and strong challenge to classical optical spectroscopy, to obtain systematic data for nearly all transitions, levels, terms and spectra. These data, even if of only moderate accuracy, are sometimes the most valuable to theoreticians. On the other hand, some modern methods are now so refined that they yield data of once quite incredible accuracy $\left(10^{-10}\right)$, so that, even 16-pole nuclear moments could be measured and reported.

Roland Winkler (Berlin Technical University)

\section{Physics of the Actinides}

The Third International Conference on the Electronic Structure of the Actinides, AN 78, which took place on the University campus of Grenoble from 30 August to 1 September, belongs to a series which started in Argonne in 1974, followed by Wroclaw in 1976. The next conference will most likely be held near Berkeley in 1981. Organized jointly by the Centre d'Etudes Nucléaires de Grenoble of the CEA and the European Institute of Transuranium Elements at Karlsruhe, AN 78 gathered together 130 participants from 14 countries (mainly European).

The scientific programme was divided into six sessions, each corresponding to one half day and consisting of two invited talks and poster communications. The themes of the sessions indicate the ground covered:

1. Physical properties of metallic magnetic compounds, 5f-band approach:

2. Physical properties of metallic magnetic compounds, 5f-localized approach;

3. Sample preparation and characterization for physical measurements;

4. Physical properties of metals and non-magnetic alloys;

5. Physical properties of ions, ionic and semi-conducting compounds;

6. Correlations between electronic structure, thermodynamics and crystal structure.

Three panel discussions were also

held upon the following topics:

Aspects of the $5 f$ electrons (de) localization;

Availability and sample preparation; limits of solid state studies on actinides

"NACl" structure actinide compounds.

In this conference centred on the condensed matter physics of the ac- tinides, great importance was attached to the chemistry. This is due to a number of factors. First, because the transuranium elements do not exist in nature and have to be prepared through nuclear reactions (mainly in neutron reactors), weighable amounts of actinides are available only up to einsteinium, which sets the limit for solid state studies. Only $10^{10}$ atoms of the next actinide, fermium, are available. Second, the preparation and characterization of actinide compounds necessitate, apart from special equipment found only in highly radioactive laboratories, the use of techniques adapted to the high cost and scarcity of the actinide materials which increases progressively as we go to the right of the actinide series: $\mathrm{kg}$ quantities of ${ }^{244} \mathrm{Cm}, \mathrm{g}$ quantities of ${ }^{252} \mathrm{Cf}$; mg quantities of ${ }^{253} \mathrm{Es},{ }^{249} \mathrm{Cf}$, ${ }^{248} \mathrm{Cm}$, total in the world. Third, it appears that for many experimental techniques (magnetization, transport, optical, X ray, neutron), single crystals are not just very useful but often necessary. These are precisely the techniques from amongst which we hope to see major advances in actinide physics.

From an experimental viewpoint indeed, the availability of sufficiently large uranium single crystals has been the stimulus for many of the detailed studies presented. This is especially true for the "NACl" type compounds, where neutron studies have emerged as a major technique in the actinide field. Both neutron and magnetic measurements revealed the importance of anisotropy effects, and gave valuable insight into the valency and spatial character of $5 f$ electrons (localized versus itinerant).

From the theoretical viewpoint, new results have been presented on the electronic structure of actinides arriv- ed at mainly through band structure calculations; the complex $\alpha$-phase of uranium has been attacked successfully. Attempts have been made also to utilize more physically transparent methods, one result being the calculation of bulk quantities such as the atomic volume of actinide pure metals. The bonding contribution from $5 f$ electrons was shown to be prominent in the beginning of the actinide series, in agreement with their complex crystallographic structure.

For the future, two conclusions can be drawn from the Conference:

- The extension of single crystal preparation for transuranium compounds is a major need for further progress to be made; AN 78 remained essentially a "Uranium" conference.

- Apart from a foreseeable development of neutron experiments, optical experiments (especially photoemission techniques) should develop and give some contribution to the still open subject of $5 f$ electrons (de) localization.

\section{Belakhovsky}

(Centre d'Etudes Nucléaires, Grenoble)

\section{UNIVERSITY OF GENEVA}

A post-doctoral research appointment is available at the Laboratory for Applied Chemistry for a solid state physicist or solid state chemist interested in joining an experimental programme (Swiss National Science Foundation) on the synthesis and the properties of ferroelectric and related magnetic materials. A three years' programme is foreseen. Renewal of this appointment is possible from year to year.

Applicants should submit résumé publication list and names of two referees to: Prof. H. Schmid, Applied Chemistry Laboratory, University of Geneva, 30, Quai ErnestAnsermet, CH-1211 Geneva 4. 